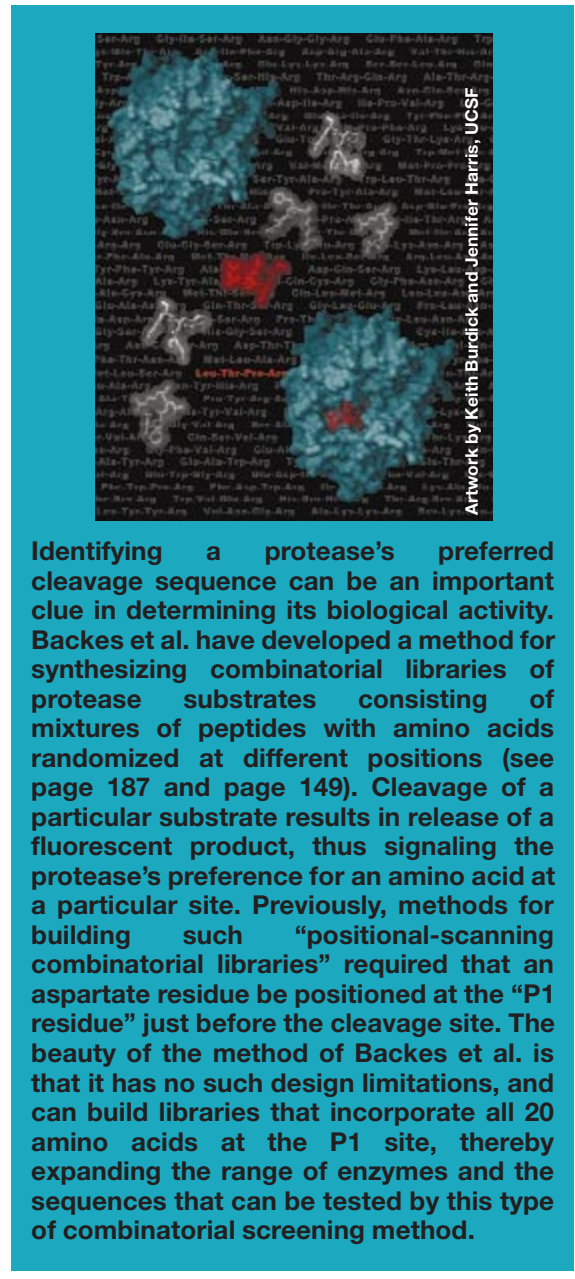

\section{A new way to integrate}

Adenovirus is a widely studied vector for gene therapy. Although the virus can be produced at high titers and directs efficient transduction and gene expression in vivo, the latter is only transient in nature. This is due in part to the episomal nature of the vector and has led to attempts to engineer an integration function into the viral genome. In this issue, Baum et al. (pp. 176 and 150) describe a modified adenoviral vector containing the long terminal repeat elements (LTRs) of the Moloney murine leukemia virus (MoMLV). In stark contrast to earlier studies, these workers show that this hybrid virus could efficiently integrate into the chromosome of recipient cells in the absence of retroviral structural proteins. These findings suggest that the hybrid virus is integrating by a nonstandard mechanism that might prove useful for future vector development.

Research Briefs written by Natalie DeWitt and Robert Frederickson

\section{Mass spec and "quantum" mechanics}

Mass spectrometry is a powerful technology to identify the proteins and peptides within biological samples. In this issue, Sweedler and colleagues demonstrate a new sampling procedure that, when used with matrixassisted laser desorption/ionization time-offlight mass spectrometry (MALDI-TOF MS), permits the identification of peptides within a single organelle (see p. 172). Their procedure required only attoliter-sized samples and allowed them to identify peptides colocalized within individual secretory vesicles of the exocrine atrial gland of the California sea hare (Aplysia californica), a large sea mollusk. The ability to identify increasingly minute quanta of peptide information by MS should find application in the proteomic analysis of neuronal processes, small cells, and individual organelles.

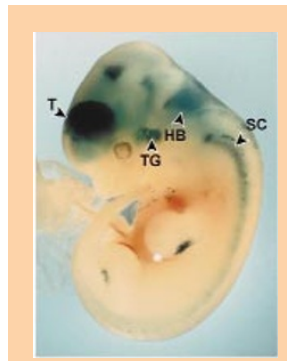

Before too long, the complete nucleotide sequence of a handful of vertebrate genomes will become available. Comparative sequence analyses should allow us to trace the molecular evolution of conserved genetic elements, such as coding regions, promoters, and repeated sequence elements. In this issue, Göttgens et al. have analyzed sequence conservation between the SCL loci of human, mouse, and chicken, which encodes a highly conserved transcription factor (see pp. 181 and 148). This analysis allowed them to identify a new neural enhancer element, which they confirmed in a transgenic Xenopus reporter assay. This work provides a simple paradigm for easy and efficient comparative sequence analyses to identify developmentally important enhancer elements.

In this issue, Westin and colleagues describe a new method for simultaneous amplification and detection of multiple targets on a single microchip array. They used an electric field to position different oligonucleotides at discrete areas on the microelectronic array, thus preventing nonspecific interactions from occurring between the primer sets. To amplify the target DNA, they used strand displacement amplification (SDA), an isothermal reaction that results in total displacement of the target strand after each amplification reaction. Combining electronic addressing with SDA provides a convenient and flexible platform for a variety of nucleic acid-based assays.

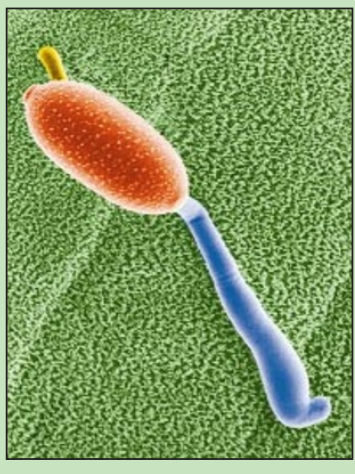

Powdery mildew fungi are ubiquitous agents of plant disease that cause greater losses to crop yields than any other single plant disease. Whereas the development of control strategies for powdery mildew is of key interest to the agricultural industry, tools for its study are relatively poor. The fungus is an obligate parasite and has not been cultured or genetically manipulated. In this issue, Spanu and colleagues report a breakthrough-the first instance of DNA-mediated transformation of Erysiphe graminis, the causative agent of barley powdery mildew (see page 205 and 146). To develop a selection marker, they isolated the E. graminis tubulin gene and derived an allele conferring resistance to the fungicide benomyl. Using biolistics to introduce DNA for this marker into the fungus as it grew on barley plants, that showed that the transformed fungus. Transformation was stable for over 20 generations, even when the fungi were grown under nonselective conditions.

\section{Phytoremediation of organic mercury}

In this issue, Meagher and colleagues show that genetically engineered plants can be used to detoxify a hazardous form of mercury that contaminates wetlands and coastal sediments (see page 213). Certain aquatic bacteria convert ionic mercury to methylmercury, an organic derivative that is more readily absorbed into plankton and the gastrointestinal tracts of fish, leading to accumulation in the food chain. Meagher and colleagues set out to generate plants capable of converting methylmercury to a less hazardous volatile form by expressing two bacterial genes that have evolved in mercury-resistant bacteria. Transgenic Arabidopsis expressing these two genes were able to extract methylmercury from their culture media and convert it sequentially to ionic mercury and then volatile elemental mercury, which the plants then transpired to the atmosphere, where it could be diluted to trace levels. 